\title{
Time synchronization of Baikal-GVD clusters
}

\author{
V.A. Allakhverdyan ${ }^{1}$, A.D. Avrorin², A.V. Avrorin², V.M. Aynutdinov²,*, R. Bannasch ${ }^{3}$, Z. \\ Bardačová4, I.A. Belolaptikov ${ }^{1}$, I.V. Borina ${ }^{1}$, V.B. Brudanin ${ }^{1}$, N.M. Budnev ${ }^{5}$, V.Y. Dik ${ }^{1}$, \\ G.V. Domogatsky², A.A. Doroshenko², R. Dvornický1,4, A.N. Dyachok ${ }^{5}$, Zh.-A.M. \\ Dzhilkibaev $^{2}$, E. Eckerová ${ }^{4}$, T.V. Elzhov ${ }^{1}$, L. Fajt ${ }^{6}$, S.V. Fialkovsky ${ }^{7}$, A.R. Gafarov ${ }^{5}$, K.V. \\ Golubkov $^{2}$, N.S. Gorshkov' ${ }^{1}$, T.I. Gress ${ }^{5}$, M.S. Katulin', K.G. Kebkal ${ }^{3}$, O.G. Kebkal ${ }^{3}$, \\ E.V. Khramov ${ }^{1}$, M.M. Kolbin ${ }^{1}$, K.V. Konischev ${ }^{1}$, K.A. Kopański ${ }^{8}$, A.V. Korobchenko ${ }^{1}$, A.P. \\ Koshechkin$^{2}$, V.A. Kozhin ${ }^{9}$, M.V. Kruglov', M.K. Kryukov², V.F. Kulepov ${ }^{7}$, Pa. Malecki ${ }^{8}$, \\ Y.M. Malyshkin ${ }^{1}$, M.B. Milenin ${ }^{2}$, R.R. Mirgazov ${ }^{5}$, D.V. Naumov ${ }^{1}$, V. Nazari ${ }^{1}$, W. Noga ${ }^{8}$, \\ D.P. Petukhov ${ }^{2}$, E.N. Pliskovsky ${ }^{1}$, M.I. Rozanov ${ }^{10}$, V.D. Rushay ${ }^{1}$, E.V. Ryabov ${ }^{5}$, G.B. \\ Safronov $^{2}$, B.A. Shaybonov ${ }^{1}$, M.D. Shelepov ${ }^{2}$, F. Šimkovic ${ }^{1,4,6}$, A.E. Sirenko ${ }^{1}$, A.V. \\ Skurikhin $^{9}$, A.G. Solovjev ${ }^{1}$, M.N. Sorokovikov ${ }^{1}$, I. Štekl ${ }^{6}$, A.P. Stromakov ${ }^{2}$, E.O. \\ Sushenok ${ }^{1}$, O.V. Suvorova ${ }^{2}$, V.A. Tabolenko ${ }^{5}$, B.A. Tarashansky ${ }^{5}$, Y.V. Yablokova ${ }^{1}$, S.A. \\ Yakovlev $^{3}$, and D.N. Zaborov ${ }^{2}$ \\ ${ }^{1}$ Joint Institute for Nuclear Research, Dubna, Russia, 141980 \\ ${ }^{2}$ Institute for Nuclear Research, Russian Academy of Sciences, Moscow, Russia, 117312 \\ ${ }^{3}$ EvoLogics GmbH, Berlin, Germany, 13355 \\ ${ }^{4}$ Comenius University, Bratislava, Slovakia, 81499 \\ ${ }^{5}$ Irkutsk State University, Irkutsk, Russia, 664003 \\ ${ }^{6}$ Czech Technical University in Prague, Prague, Czech Republic, 16000 \\ ${ }^{7}$ Nizhny Novgorod State Technical University, Nizhny Novgorod, Russia, 603950 \\ ${ }^{8}$ Institute of Nuclear Physics of Polish Academy of Sciences (IFJ PAN), Krakow, Poland, 60179 \\ ${ }^{9}$ Skobeltsyn Institute of Nuclear Physics MSU, Moscow, Russia, 119991 \\ ${ }^{10}$ St. Petersburg State Marine Technical University, St. Petersburg, Russia, 190008 \\ ${ }^{\star}$ E-mail: aynutdin@yandex.ru
}

Baikal-GVD neutrino telescope is under construction now. The 2021 telescope configuration includes 8 clusters of 288 photodetectors each. Cluster is a functionally complete detector that can register events, muons and cascade showers, in stand-alone mode and jointly with other clusters. The angular resolution of the detector is determined by the accuracy of the time measurement with the telescope channels. The uncertainty of the time measurement depends, in particular, on the accuracy of the time synchronization of the channels. The article describes the method of synchronization of Baikal-GVD and presents the results of studies of the accuracy of time synchronization both for individual clusters and for the installation as a whole.

37th International Cosmic Ray Conference (ICRC 2021)

July 12th-23rd, 2021

Online-Berlin, Germany

\section{"Presenter}

(c) Copyright owned by the author(s) under the terms of the Creative Commons 


\section{Introduction}

Currently, the construction of the Baikal-GVD neutrino telescope is underway in Lake Baikal [1,2]. The 2021 telescope configuration includes 8 clusters of 288 photodetectors each. The photodetectors form a spatial structure located at a lake depth of about one kilometer, designed to register the Cherenkov radiation of charged particles formed as a result of the interaction of neutrinos. Each cluster is a functionally complete detector that can register events, muons, and cascade showers, either as stand-alone detector or jointly with other clusters. The angular resolution of the detector is determined by the accuracy of measuring the arrival time of Cherenkov photons by the telescope channels. The accuracy of the time measurement is determined by the time resolution of the photodetectors, by uncertainties in the time calibration of the channels, and a number of other factors. Fig. 1.1 presents angular resolution of reconstructed tracks at Baikal-GVD as a function of true muon energy with a different time resolution of the measuring channels. The error in the measurement of time associated with the photodetectors and channels calibration was fixed at $2 \mathrm{~ns}$, whereas the accuracy of the time synchronization of channels was varied from 0 to $20 \mathrm{~ns}$.

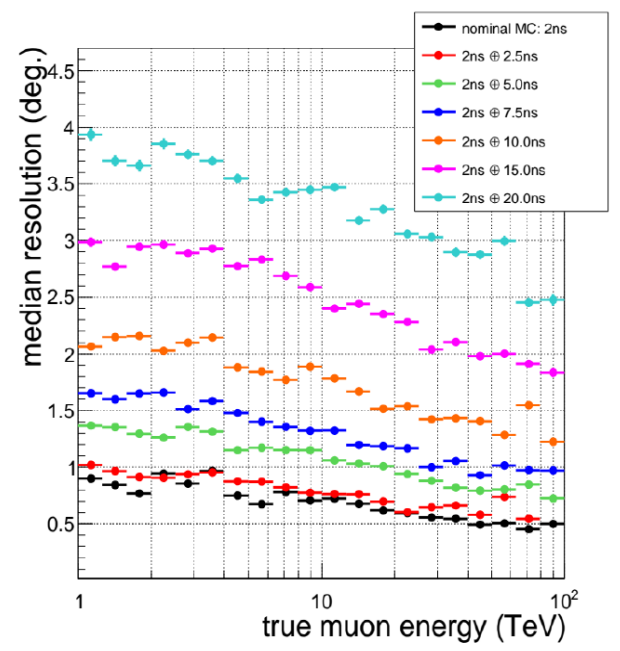

Fig. 1.1. Dependence of the angular resolution of the Baikal-GVD cluster (using $\chi^{2}$-based track reconstruction algorithm) on the accuracy of the time measurement by the telescope channels.

This article is devoted to the issues of time synchronization of Baikal-GVD channels. It describes the synchronization methods and presents the results of experimental studies of the accuracy of channels synchronization for both individual clusters and the installation as a whole.

\section{Time synchronization system}

The Cherenkov radiation of charged particles in the Baikal-GVD is detected using optical modules (OMs), with the photomultiplier tubes (PMT) as its photosensitive element $[3,4]$. The main structural element of the Baikal-GVD data acquisition system is the section of optical modules [5], which includes 12 OMs. A total of three sections placed on a supporting vertical cable form a string. The distance between the OMs in a string is $15 \mathrm{~m}$, the instrumented length of the string is $525 \mathrm{~m}$. There are eight strings grouped into a cluster. The data acquisition center of the cluster is connected to the shore station by an optical hybrid cable with a length of $6-7 \mathrm{~km}$ (depending on the location of the cluster in relation to the shore). Each cluster consists of a total of $288 \mathrm{OMs}$, which form the measuring channels of the detector. The channels allow to measure 
the time and charges of pulses coming from OMs. The Baikal-GVD time synchronization system provides the operation of all the channels in a single time scale. It includes two subsystems that provide synchronization of channels within a single cluster, and synchronization of clusters with each other. The work of these subsystems is based on different principles. Synchronization of channels within a single cluster is carried out by means of a common trigger signal received by all its sections. To synchronize the clusters with each other (inter-cluster synchronization), a single clock frequency is used, formed by the equipment of the shore station and transmitted to all clusters of the installation. This approach is largely due to the technical features of the underwater cable system of the telescope. Hybrid cables connecting the clusters to the Shore station comprise fiber-optic channels that allow reliable transmission of clock frequencies at $100 \mathrm{MHz}$ or more over long distances. Cable communications within clusters are made without the use of optical fiber, and the transmission of high clock frequency over them is difficult.

Fig. 2.1 shows a diagram of the organization of the Baikal-GVD synchronization system. The signals from the twelve optical modules of each section are sent to the ADC unit of the Master, forming 12 measuring channels. The Master generates a section request signal, the condition for the formation of which is the coincidence of signals from two adjacent OMs in a time window of $100 \mathrm{~ns}$. The request signals from all sections of the string are combined in the string trigger module, forming a string request. String requests, in turn, are sent to the central trigger module of the cluster, which forms a common trigger. The time of formation of a common trigger (time of the event) is measured using inter-cluster synchronization units. The common trigger is passed to the string modules, where it branches into three sections and enters to the Masters. Trigger signals initiate the process of forming of the time frames for the channels, which contain information about the shape of the optical module pulses. A set of time frames from the sections of the cluster initiated by the common trigger makes up a physical event.

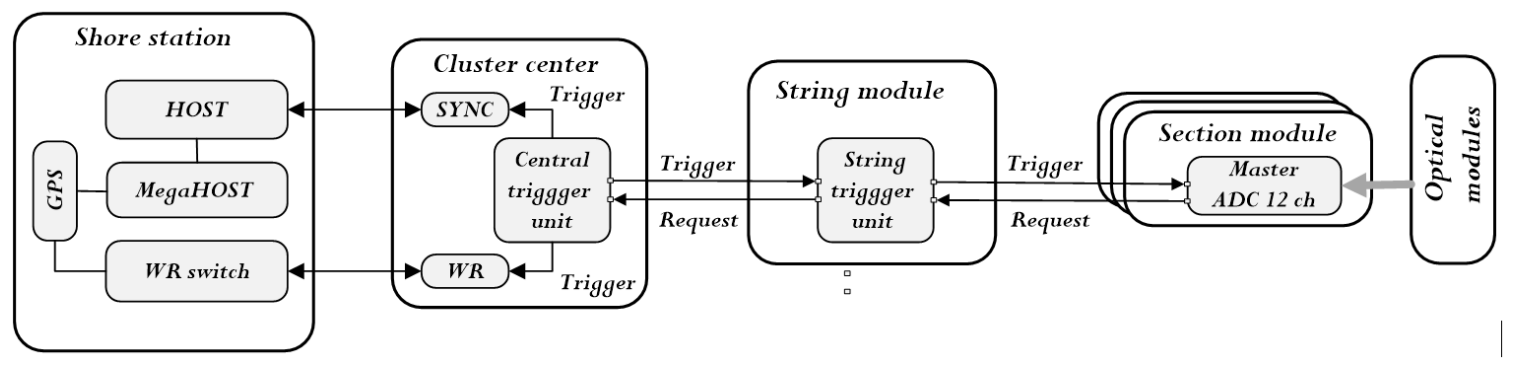

Fig. 2.1. Block diagram of the Baikal-GVD synchronization system.

The accuracy of channels synchronization is determined by the specific of request and trigger generation by the Master unit. The leading edge of these signals is tied to the clock frequency of the electronics units $(200 \mathrm{MHz})$, which leads to a 5-nanosecond uncertainty of their formation time. This does not introduce errors when measuring the time difference of the channels of one section, but affects the registration of pulses by different sections of the cluster. The trigger units of the strings (see Fig. 2.1) do not cause additional time uncertainty, since for them the time of output signals is tied to the input one. Taking into account the specifications of the functioning of the trigger system, it is possible to estimate the expected accuracy of measuring the time difference $\Delta \mathrm{t}$ by the cluster channels. For channels of one section, the measuring accuracy $\Delta \mathrm{t}$ must be less than $1 \mathrm{~ns}$. This accuracy is achieved by interpolating the pulse shape measured by the ADC with a sampling rate of $5 \mathrm{~ns}$. For channels of different sections, the error $\Delta \mathrm{t}$ consists of the time 
uncertainties of the moments when the trigger signals are fixed by the Master unit of the section. The expected standard deviation of $\Delta \mathrm{t}$ is $\sim 2 \mathrm{~ns}$.

To synchronize the operation of clusters, the time of the leading edge of a common trigger is measured on each of them (see Fig. 2.1). The clocks of all clusters are synchronized from a single clock frequency source, the signals from which are transmitted via fiber-optic lines from the shore station. The measurement is carried out by two independent systems: WR (White Rabbit) and SSBT (Synchronization System of Baikal neutrino Telescope). The WR synchronization system [6] was developed by the European Organization for Nuclear Research (CERN) and is an array of specialized devices integrated into an optical network with an Ethernet transmission protocol and a speed of $1 \mathrm{Gbit} / \mathrm{s}$. The WR system allows the transmission of data and synchronization to several thousand nodes at distances of about $10 \mathrm{~km}$ with sub-nanosecond accuracy.

The SSBT system was specially designed for the Baikal-GVD project in order to develop further the data acquisition system of the clusters based on fiber-optic technologies. The SSBT includes three types of electronic units: the $S Y N C$ time measurement unit located in the center of the cluster, and the HOST and MegaHOST synchro-units located at the shore station. The SYNC unit provides a time measurement of the common trigger. The principle of operation of $S Y N C$ and the Master units is almost the same. The SYNC records the time of a trigger with $5 \mathrm{~ns}$ discretization and forms a packet of information that is included in the overall data flow of the cluster. The single clock frequency for the SYNC of all clusters comes from the onshore HOST units. In addition to the clock frequency, the HOST sends timestamps to SYNC to synchronize the local clock start and to measure the time of the signals passing through the fiber-optic cables connecting them (the optical length of the cable). To measure the optical length, the timestamp received by SYNC is sent back to the HOST, which measures the delay between the sent and received timestamp. The optical length measurement accuracy is better than $1 \mathrm{~ns}$.

The HOST allows one to synchronize up to 8 SYNC units located in eight clusters. Further expansion of the system is enabled by a MegaHOST unit which allows to combine up to 8 HOSTs. The MegaHOST generates a single clock frequency of $100 \mathrm{MHz}$ for all HOSTs, which ensures synchronous operation of the local clock of all clusters of the installation. The MegaHOST clock, in turn, is synchronized with an external GPS / GLONASS receiver with an accuracy of $15 \mathrm{~ns}$ UTC. The time of the MegaHOST local clock and the time received from the GPS can be read through the control channel.

\section{Study of synchronization accuracy}

To study the characteristics of the synchronization system of the telescope a series of calibration measurements were analyzed which included simultaneous illumination of several clusters by a laser light source. The lasers are installed between clusters for mutual calibration of their channels. The wavelength of the laser sources is $532 \mathrm{~nm}$, the flash duration is $1 \mathrm{~ns}$, and the maximum radiation intensity is about $10^{15}$ photons. The laser beam is introduced through an optical waveguide into a diffuser, which works as a point quasi-isotropic light source (see Fig. 3.1). Lasers and the electronics controlling their operation are mounted on a load-carrying vertical cable, forming laser stations. At one station, two lasers are placed at different depths of the lake. Laser stations are placed between clusters at a distance of 150 meters from their central strings. The placement of lasers in the installation and an example of a laser calibration event are shown 
in Fig. 3.1. The color of the triggered channels in the figure indicates the time of registration: from red (early signals) to blue (late one).
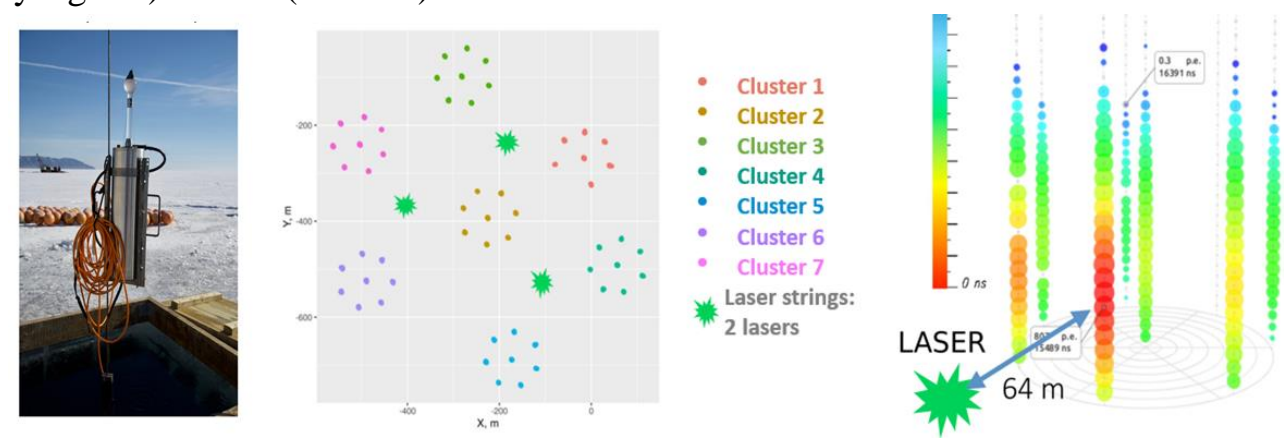

Fig. 3.1. A laser source with a light diffuser (left), the position of clusters and laser stations (center), and an example of a laser event (right).

To evaluate the accuracy of the synchronization system, the data obtained during the calibration series of the laser located between clusters 4 and 5 was used (26.06.2020). The accuracy of the synchronization is quantified with the RMS of the distribution of the difference in response time $\Delta t$ between any two channels of the clusters. For the analysis, channels were selected for which the average charge of the laser signals exceeded 10 photoelectrons to reduce the statistical error associated with PMT jitter $(\sim 3$ ns under single-photoelectron illumination conditions). Fig. 3.2 shows the distribution of channels on RMS of $\Delta \mathrm{t}$.
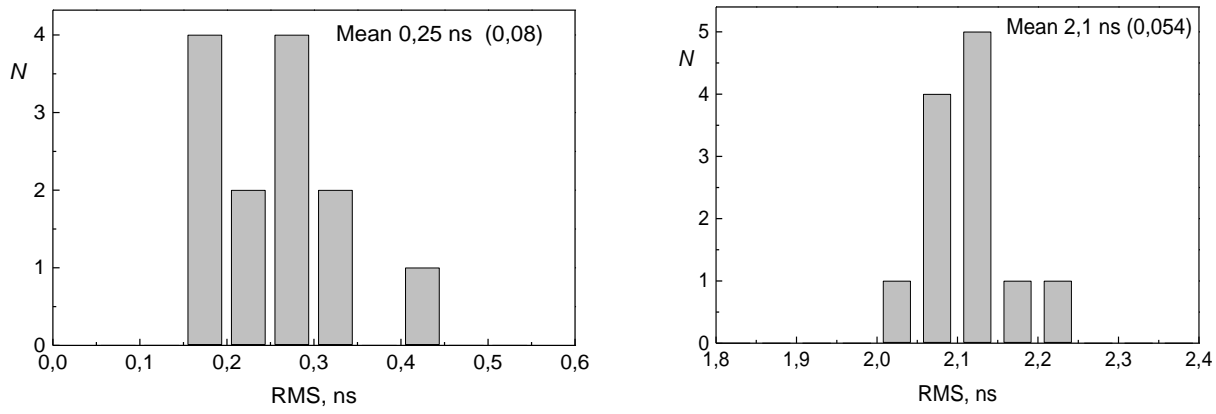

Fig. 3.2. Distribution of channel pairs on the RMS of the measured time difference: channels of one section (left) and channels of different sections (right).

The left panel of Fig. 3.2 shows that the synchronization of the channels of one section of the OMs (single 12-channel ADC board) occurs with an accuracy of better than $1 \mathrm{~ns}$. The obtained value of RMS of 0.25 ns characterizes the accuracy of the estimation of the response time of the channels using the interpolation of the pulse shape from the optical modules. The right panel of Fig. 3.2 shows a similar distribution obtained for channels located on different sections of the cluster and serviced by different Master units (two different ADC boards). As mentioned in the section 2, the Master units capture the trigger signal that performs the synchronization function with a $5 \mathrm{~ns}$ discreteness. Under these conditions, the measured average RMS was $2.1 \pm$ $0.05 \mathrm{~ns}$, which is in good agreement with the expected value of $2.04 \mathrm{~ns}$. To assess the accuracy of inter-cluster synchronization, the RMS of the difference in the event times $d T$ ( $T$ is the time of formation of the cluster trigger), was estimated for WR and SSBT systems. These results are shown in Fig. 3.3. 


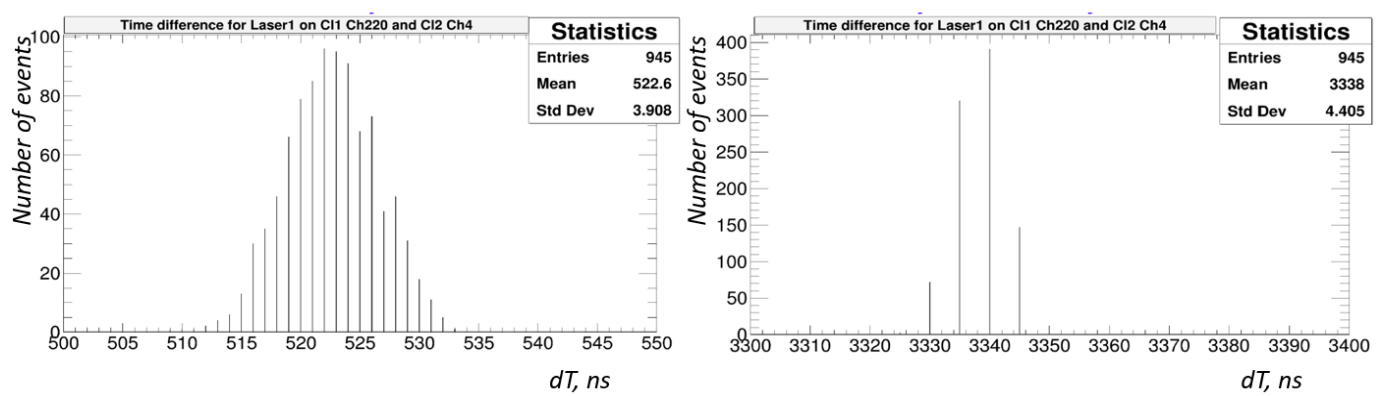

Fig. 3.3. Distribution of the laser events on the difference in their time $d T$, measured on the cluster 4 and 5 by two independent synchronization systems: WR (left) and SSBT (right).

The accuracy of inter-cluster synchronization is determined by the time uncertainties that occur when generating the request and trigger signals in the sections and in the cluster centers. The measured RMS value of the difference in the time of event registration on the two clusters for $W R$ was $3.9 \mathrm{~ns}$, which is in satisfactory agreement with the expected value of $3.5 \mathrm{~ns}$. For SSBT, this value is expected to be higher and is $4.4 \mathrm{~ns}$. This is due to the additional uncertainty that occurs when the $S Y N C$ units detect the triggers.

\section{Conclusion}

Studies of the Baikal-GVD synchronization system with a laser calibration light source showed the correctness of its operation and allowed us to evaluate the synchronization accuracy for all structural elements of the telescope data acquisition system. The results obtained are in good agreement with the expectation. The accuracy of channel synchronization within a single telescope section is significantly better than $1 \mathrm{~ns}$. For different sections of the cluster, this accuracy is about $2 \mathrm{~ns}$. At this level of accuracy, the cluster synchronization system does not affect the angular resolution of the Baikal-GVD cluster. The results of measurements of the synchronization accuracy of two clusters performed by two independent systems are consistent with each other and give the accuracy of events synchronization at the level of 4 ns. Taking into account the significant distance between the cluster centers (200 - 300 meters), it is not expected that an additional error of $4 \mathrm{~ns}$ will affect the angular accuracy of the telescope significantly.

The work was partially supported by RFBR grants 20-02-00400 and 19-29-11029.

\section{References}

[1] I.A. Belolaptikov et al. (Baikal Collaboration), The Baikal underwater neutrino telescope: Design, performance, and first results, Astropart. Phys. 7 (1997) 263.

[2] A.V. Avrorin et al. (Baikal Collaboration), The Gigaton volume detector in lake Baikal, Nucl. Instr. and Meth. in Phys. Res. A, 639 (2011) 30.

[3] A.D. Avrorin et al. (Baikal Collaboration), The optical module of Baikal-GVD, EPJ Web of Conferences, 116 (2016) 01003.

[4] A.D. Avrorin et al. (Baikal Collaboration), The optical detection unit for Baikal-GVD neutrino telescope, EPJ Web of Conferences, 121 (2016) 05008.

[5] V.M. Aynutdinov et al. (Baikal Collaboration), The data acquisition system for Baikal-GVD, EPJ Web of Conferences, 116 (2016) 5004.

[6] J. Serrano et al, The White Rabbit Project, Proceedings of ICALEPS 2009, ICALEPCS

TUC004 (2009) http://accelconf. web.cern.ch/accelconf/icalepcs2009/papers/tuc004.pdf 